\title{
Effects of Nigella sativa seeds and certain species of fungi extracts on number and activation of dural mast cells in rats
}

\author{
E Kilinc ${ }^{1}$, Y Dagistan², B Kotan ${ }^{3}$, A Cetinkaya ${ }^{4}$ \\ ${ }^{1}$ Department of Physiology, Faculty of Medicine, Abant Izzet Baysal University, Bolu, Turkey \\ ${ }^{2}$ Department of Neurosurgery, Faculty of Medicine, Abant Izzet Baysal University, Bolu, Turkey \\ ${ }^{3}$ Department of Geographical Sciences, Bolu Science High School, Bolu, Turkey \\ ${ }^{4}$ Department of Experimental Animal Application and Research Center, Abant Izzet Baysal University, Bolu, Turkey
}

Received: June 28, 2016

Accepted: November 14, 2016

\begin{abstract}
In this study, we aimed to investigate the effects of Nigella sativa seeds and certain species of fungi extracts on the number and degranulation states of dural mast cells in rats. Rats were fed ad libitum with normal tap water or tap water with extract of $N$. sativa seed, Ramaria condensata, Lactarius salmonicolor, Lactarius piperatus, and Tricholoma terreum for 3 days. Mast cells in dura mater were counted and evaluated in terms of granulation and degranulation states. Compound 48/80, a mast cell degranulating agent, and T. terreum significantly increased the percent of degranulated mast cells in dura mater, respectively $(p<0.01$ and $p<0.05)$. Moreover, T. terreum causes a significant increase in the total number of mast cells $(p<0.05)$. $N$. sativa significantly inhibited mast cell degranulation induced by both the compound 48/80 and T. terreum $(p<0.05)$, and significantly decreased the mast cell numbers increased by T. terreum $(p<0.05)$. Our results suggested that $T$. terreum following ingestion can contribute to headaches like migraine via dural mast cell degranulation and $N$. sativa may be able to exert analgesic and anti-inflammatory effects by stabilizing dural mast cells. However, investigation is needed to determine the ingredients of $N$. sativa that may be responsible for these beneficial effects.
\end{abstract}

Keywords: migraine, neurogenic inflammation, dura mater, mast cell, Nigella sativa, Tricholoma terreum

\section{Introduction}

Mast cells are multifunctional paracrine cells originated from pluripotent progenitor cells in the bone marrow (12). They are found in almost all organs throughout the body, in particular, abundantly in cranial dura mater and placed very close to blood vessels and nerves. Mast cells are known as effector cells of allergic inflammation, but they also play a key role in physiological processes, such as immune responses, and in pathophysiological processes, such as migraine (15). Mast cells express many vasoactive, nociceptive, and proinflammatory molecules, such as substance-P (SP), calcitonin gene-related peptide (CGRP), histamine, and interleukin 6 (IL-6), in response to various stimulus (21). These mediators are stored in the mast cell granules. When mast cells are activated, the mediators spread to their resident environment and affect vascular and neural functions. Migraine is one of the most common neurological diseases, characterized by recurrent unilateral headache, nausea, vomiting,

\footnotetext{
Corresponding author: Erkan Kilinc

Department of Physiology, Faculty of Medicine, Abant Izzet Baysal University

Bolu 14280, Turkey

Phone: +90 05331380483; Fax: +90 37425345 59; E-mail: e_kilinc_27@hotmail.com
} 
photophobia, and phonophobia (15). Migraine pathophysiology is still not completely understood although many investigations about the mechanism have been carried out. Recent studies have suggested that sterile neurogenic inflammation in the dura mater, caused by trigeminal nociceptive activation, is responsible for the pathophysiology of migraine (14). It was shown that dural mast cell degranulation led to prolonged activation of meningeal nociceptors in the trigeminal ganglion (15). Interactions among dural mast cells, trigeminal sensory nerve, and dural blood vessels play a key role in the neurogenic inflammation component of the migraine pathophysiology. Antigens of certain species of fungi, following their ingestion, cause IgE-mediated degranulation of mast cells by increasing IgE release in the body (17).

Some species of wild edible fungi including Ramaria condensata, Lactarius salmonicolor, Lactarius piperatus, and Tricholoma terreum grow wildly in Bolu, Turkey. Most people who ingested these fungi species go to the emergency department of hospitals with complaints including headache and vomiting. It was reported that some of these fungi have antioxidant (T. terreum, L. piperatus, and L. salmonicolor), antimicrobial (T. terreum and $L$. piperatus), and anticancer $(T$. terreum $)$ properties $(4,16,18)$. However, the effects and mechanisms of these fungi species on headache have not been elucidated to date.

Nigella sativa is an annual plant, known as black cumin, and its black seeds are commonly used as a spice as well as for cure of many ailments (2). It was reported that extracts, oil, especially thymoquinone, main ingredient of the volatile oil, and the other active component of $N$. sativa seeds possess antinociceptive, antihistaminic, and anti-inflammatory effects (3). It was reported that the extract of $N$. sativa seeds showed analgesic effect in mouse model of pain (2). N. sativa and thymoquinone, main ingredient of the volatile oil in N. sativa seeds, application decreased mast cell numbers and histamine levels in gastric tissue which is damaged by ethanol (13), and inhibited histamine release from peritoneal mast cells induced by the compound $48 / 80$ in rats (9). But there is no information about the effects of extract of $N$. sativa seeds on the dural mast cells. Therefore, in this study, we hypothesized that dural mast cell degranulation may be a putative mechanism for headache, which is induced by certain kinds of fungi and prevented by the extract of $N$. sativa seeds that were reported to have analgesic effects from several studies.

\section{Materials and Methods}

\section{Animals}

Forty male Wistar rats weighing 200-250 g were used in the study. All procedures applied were carried out following the guidelines for the care and use of experimental animals in Abant Izzet Baysal University with an approval from the local animal experiment research ethics committee of the university. The rats were fed ad libitum with a standard pellet diet and were housed in their cages with a 12-h light/dark cycle at $22 \pm 2{ }^{\circ} \mathrm{C}$.

\section{Groups and treatment of rats}

Rats were randomly divided into eight groups as follows: (a) rats in the control group $(n=5)$ were fed ad libitum with normal tap water for 3 days; (b) rats in the C-48/80 group $(n=5)$ were fed ad libitum with normal tap water for 3 days and injected with compound 48/80 [Sigma-Aldrich, Schnelldorf, Germany, $2 \mathrm{mg} / \mathrm{kg}$, intraperitoneal (i.p.)] at the end of the third day; (c) rats in the Nigella $+\mathrm{C}-48 / 80$ group $(n=5)$ were fed ad libitum with tap water with extract of $N$. sativa seeds $(4 \mathrm{mg} / \mathrm{ml})$ (23) for 3 days and injected with compound 
$48 / 80$ (2 mg/kg, i.p.) at the end of the third day; (d) rats in the Condensata group $(n=5)$ were fed ad libitum with tap water with extract of $R$. condensata $(4 \mathrm{ml} / \mathrm{kg})(5)$ for 3 days; (e) rats in the Salmonicolor group $(n=5)$ were fed ad libitum with tap water with extract of L. salmonicolor ( $4 \mathrm{ml} / \mathrm{kg}$ ) (5) for 3 days; (f) rats in the Piperatus group $(n=5)$ were fed ad libitum with tap water with extract of L. piperatus $(4 \mathrm{ml} / \mathrm{kg})(9)$ for 3 days; $(\mathrm{g})$ rats in the Terreum group $(n=5)$ were fed ad libitum with tap water with extract of T. terreum $(4 \mathrm{ml} / \mathrm{kg})$ (5) for 3 days; (h) because of extract of $T$. terreum caused degranulation of dural mast cells and an increase in the number of dural mast cells, we constituted also a group as Nigella + Terreum, therefore, rats in the Nigella + Terreum group $(n=5)$ were fed ad libitum with tap water with a combination of extract of $N$. sativa seeds $(4 \mathrm{mg} / \mathrm{ml})(23)$ and $T$. terreum $(4 \mathrm{ml} / \mathrm{kg})(5)$ for 3 days.

\section{Plant and fungi materials and preparation of the extracts}

The fresh extract of $N$. sativa seeds was given as a gift by Dr. Arzu from the Faculty of Agriculture and Natural Sciences, Abant Izzet Baysal University. In brief, powdered seeds of $N$. sativa were extracted in a Soxhlet extractor with ethanol as described previously (10). The $N$. sativa seeds were purchased from a local herbal shop in Bolu, Turkey, and the seeds were powdered in a mixer. The mixture of $100 \mathrm{~g}$ of powdered seeds and $350 \mathrm{ml}$ of $70 \%$ ethanol was soaked for $48 \mathrm{~h}$ at room temperature. Then, extraction solution was filtered and the solvent was removed by rotary evaporation at $40{ }^{\circ} \mathrm{C}$ under vacuum. The dried extract was dissolved in the tap water to obtain the dose of $4 \mathrm{mg} / \mathrm{ml}$ (23). Fungi including $R$. condensata, L. salmonicolor, L. piperatus, and T. terreum were collected from the land of Bolu, Turkey, and identified by an experienced biologist. The fresh extraction of the fungi was prepared as described previously by Zeynalov (24). In brief, fungi were dried in the open air under shade in the laboratory at $25^{\circ} \mathrm{C}$. The dried samples $(100 \mathrm{~g})$ were rended into small pieces and $100 \mathrm{~g}$ of rended pieces of each dried fungus was soaked in $30 \%$ ethanol for 2 weeks in capped bottles at $40^{\circ} \mathrm{C}$. Extraction solution was filtered and the solvent was removed to obtain a powder. The powdered samples were stored at $4{ }^{\circ} \mathrm{C}$. One milligram of each powder extract was dissolved in $1 \mathrm{ml}$ of the tap water to obtain the dose of $4 \mathrm{ml} / \mathrm{kg}(5)$.

Whole-mount preparations of dura mater and toluidine blue staining for mast cells in the dura mater

Rats in all groups were sacrificed by cervical dislocation under ketamine anesthesia $(60 \mathrm{mg} / \mathrm{kg}$, i.p.) at the end of the third day of the experiments, but rats in the C-48/80 and Nigella + C-48/80 groups were sacrificed at the end of the third day of the experiments $2 \mathrm{~h}$ after the injection of the compound $48 / 80$ ( $2 \mathrm{mg} / \mathrm{kg}$, i.p.). Whole-mount preparations of dura mater were prepared onto glass slides with polylysine to stain dural mast cells as described previously (14). Shortly, the head was quickly separated from the body and cleaned from skin and muscles, then the skull was cut bilaterally above the mastoid bone, and the brain was removed without harming the cranial dura mater. Skulls obtained were kept in 4\% paraformaldehyde solution for overnight. Each skull was divided into two halves along sagittal plane; dura mater was gently removed from each hemiskull and placed onto the glass slides with polylysine via a whisker. After the whole-mount preparations were dried in the open air at room temperature, they were stained with toluidine blue (pH: 2.5) to observe mast cells. Dural mast cells were counted and classified as either granulated or degranulated with a light microscope (Olympus CX21) by a blinded observer 


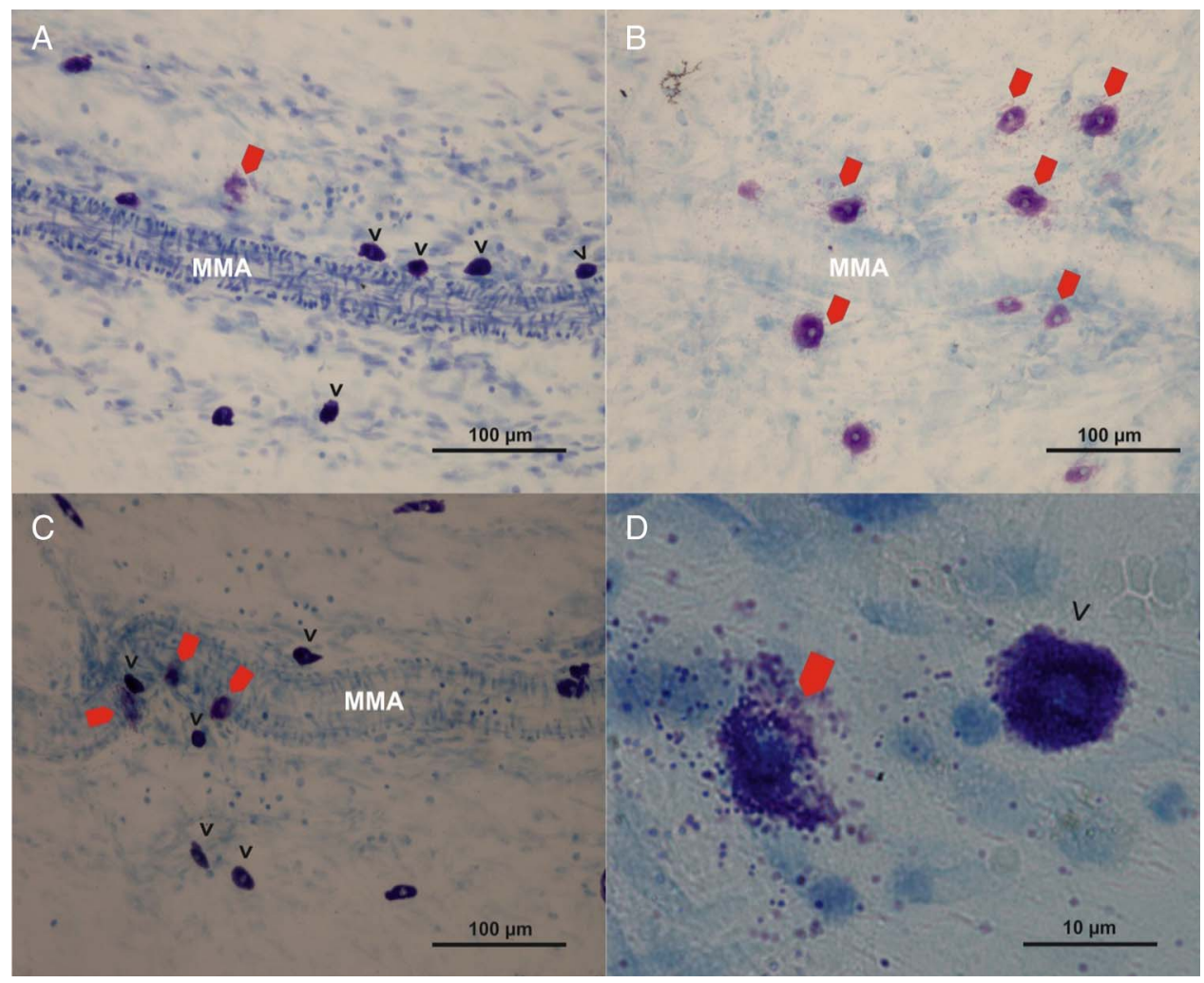

Fig. 1. The effects of $N$. sativa on degranulation states of dural mast cells induced by compound 48/80. (A) Mast cells in the control group, 20× magnification, (B) compound 48/80 induced massive degranulation of mast cells,

$20 \times$ magnification, (C) N. sativa decreased mast cell degranulation induced by compound 48/80, 20× magnification, and (D) typical intact and degranulated mast cells with $100 \times$ magnification in the Nigella + C-48/80 group. Open arrowheads show intact mast cells and solid arrowheads show degranulated mast cells. Mast cells in the dura mater are located close to the middle meningeal artery in all groups.

MMA: middle meningeal artery

with $10 \times$ magnification in the bilateral five objective areas containing the main branches of the middle meningeal artery in each dura mater. Degranulation of mast cells is a cellular process, when activated mast cells release a mixture of compounds such as histamine and serotonin from its cytoplasmic granules to the outside of the cell (Fig. 1B, D). The percent of mast cells degranulated was calculated for each rat as follows: (number of degranulated mast cells/number of total mast cells) $\times 100 \%$. Microscopic images of the dura regions used were taken with a camera (Nikon DS-Fi1, Nikon, Japan) attached to the microscope (Nikon Eclipse 80i, Nikon, Japan).

\section{Statistical analysis}

The values obtained from the experiments are expressed as the mean \pm standard error of mean. Statistical analysis was carried out using SPSS for Windows (version 17.0, SPSS Inc., Chicago, IL, USA). One-way analysis of variance followed by Dunnett's post hoc test was used to analyze percent degranulation, total mast cell numbers, and granulated or degranulated mast cell density. $p<0.05$ was considered to be statistically significant. 


\section{Results}

The percent of degranulated mast cells in the dura of rats that received compound 48/80 was significantly higher $(38.9 \pm 1.96 \%, p<0.01)$ than that in control rats $(12.6 \pm 1.2 \%, p<0.01$; Figs $2 \mathrm{~A}$ and $1 \mathrm{~A}, \mathrm{~B})$, but there was no significant difference in terms of total mast cell numbers between the control $(418 \pm 0.88, p>0.05)$ and C-48/80 (434 $\pm 1.58, p>0.05$; Fig. 2B) groups. The percent of degranulated mast cells in the dura of rats in the Nigella + C-48/80 group was significantly less $(25.1 \pm 0.93 \%, p<0.05)$ than that in the $\mathrm{C}-48 / 80$ group $(38.9 \pm 1.96 \%, p<0.05$; Figs $2 \mathrm{~A}$ and $1 \mathrm{~B}, \mathrm{C})$, but there was no significant difference in terms of total mast cell numbers between the Nigella + C-48/80 $(422 \pm 1.94, p>0.05)$ and C-48/80 ( $434 \pm 1.58, p>0.05$; Fig. 2 B $)$ groups.

The percent of degranulated mast cells in the dura of rats in the groups including Condensata $(13.7 \pm 0.99 \%, p>0.05)$, Salmonicolor $(12.5 \pm 0.69 \%, p>0.05)$, and Piperatus
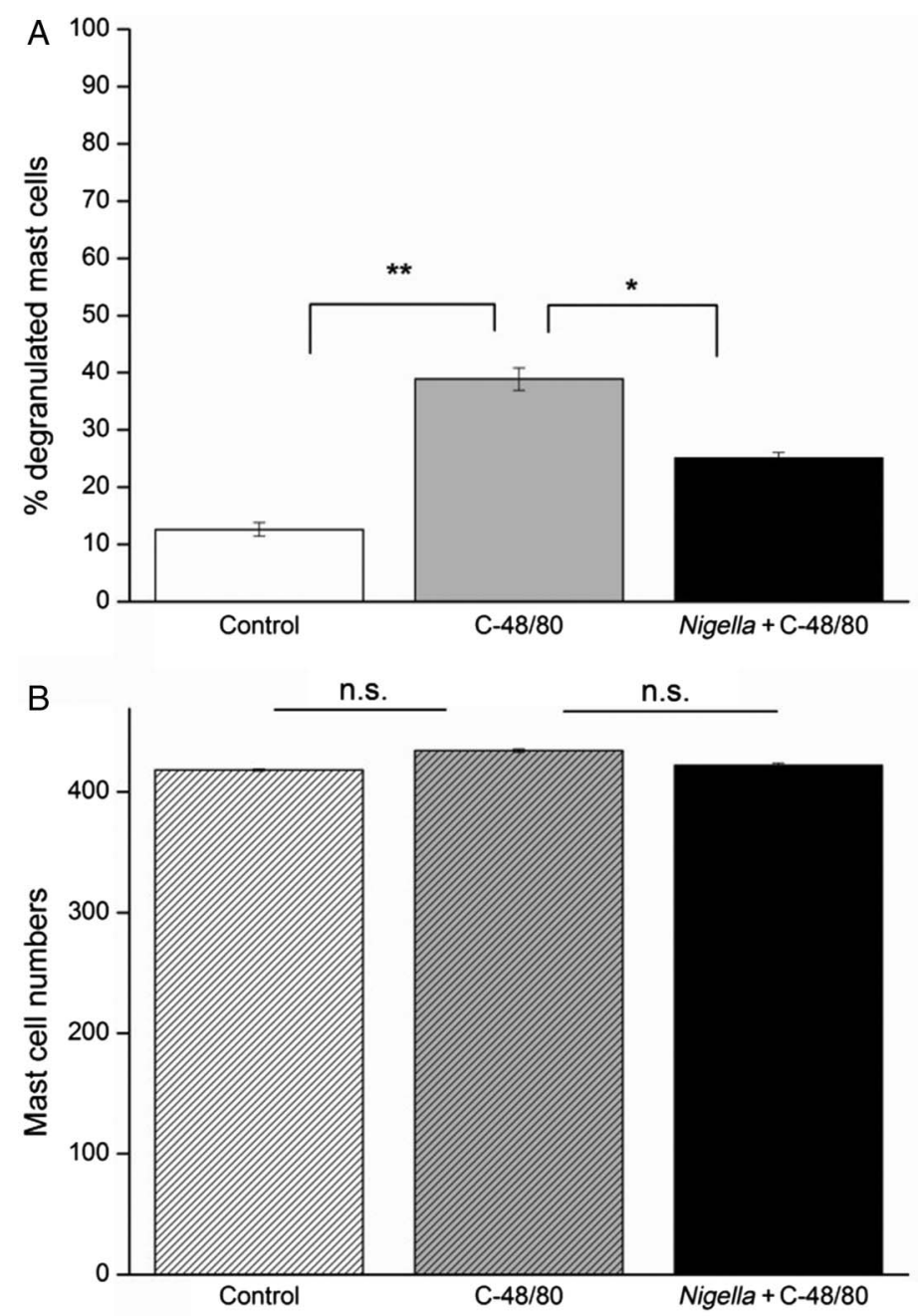

Fig. 2. N. sativa decreases mast cell degranulation induced by compound 48/80. (A) Compound $48 / 80$ increased the percent of degranulated mast cells in dura mater $(* * p<0.01)$ and $N$. sativa decreased those $\left({ }^{*} p<0.05\right)$ and (B) compound $48 / 80$ or $N$. sativa did not change total mast cell numbers in dura mater 
$(13.59 \pm 0.71 \%, p>0.05)$ did not significantly differ from the control group $(12.6 \pm 1.2 \%$, $p>0.05$; Fig. 3A), and there were no significant differences in terms of total mast cell numbers among the groups including Condensata $(457 \pm 2.96, p>0.05)$, Salmonicolor $(397 \pm 2.43, p>0.05)$, Piperatus $(412 \pm 2.09, p>0.05)$, and control $(418 \pm 0.88, p>0.05$; Fig. 3B) either.

On the other hand, the percent of degranulated mast cells in the dura of rats in the Terreum group was significantly higher $(17.95 \pm 0.57 \%, p<0.05)$ than in control rats $(12.6 \pm 1.2 \%, p<0.05$; Figs $3 \mathrm{~A}$ and $4 \mathrm{~A}, \mathrm{~B})$, likewise, total mast cell numbers in the dura mater of rats in the Terreum group were also significantly higher $(557 \pm 1.44, p<0.05)$ than in control rats $(418 \pm 0.88, p<0.05$; Figs $3 \mathrm{~B}$ and $4 \mathrm{~A}, \mathrm{~B})$.

The percent of degranulated mast cells in the dura of rats in the Nigella + Terreum group was significantly less $(12 \pm 0.92 \%, p<0.05)$ than in rats in the Terreum group
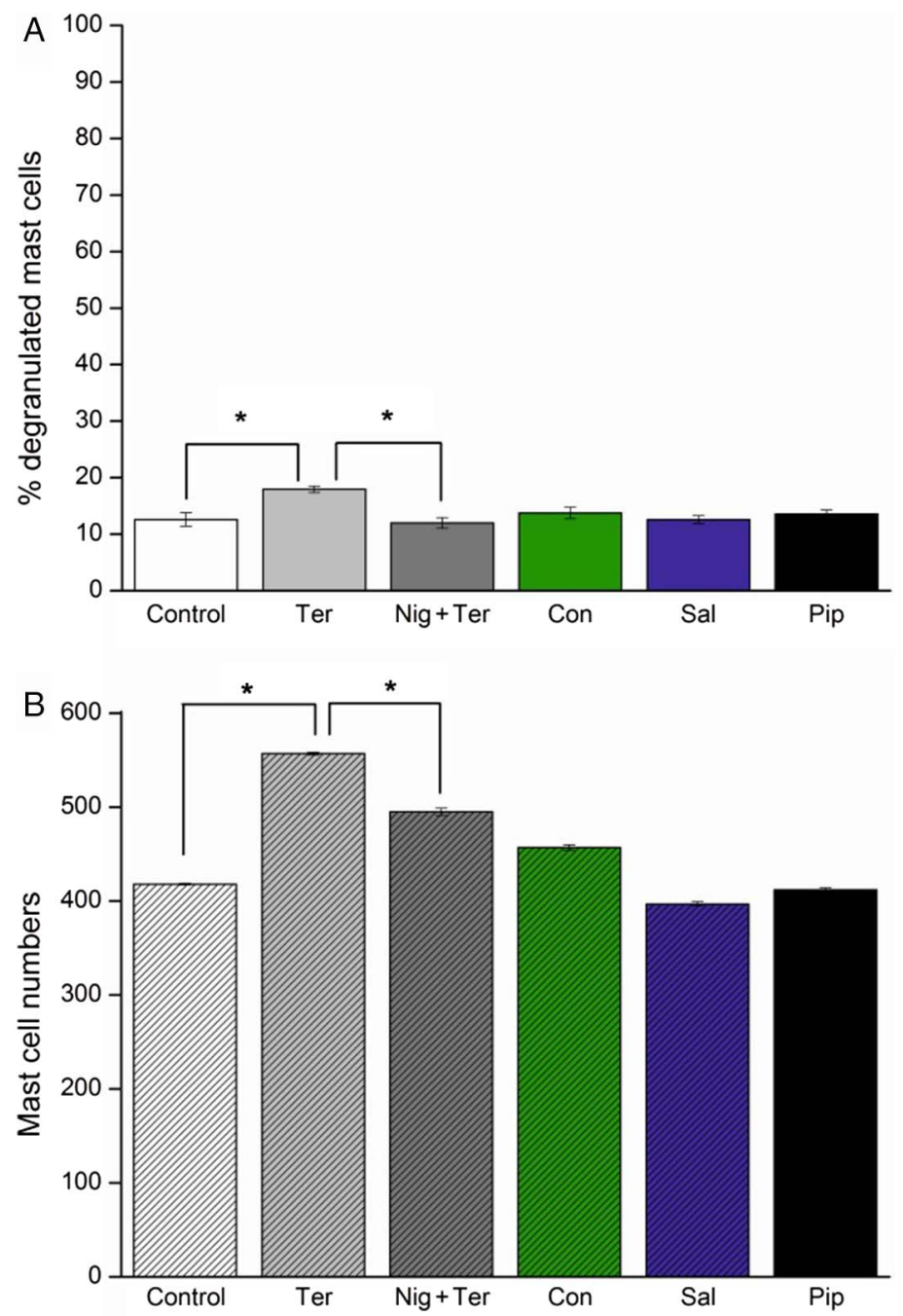

Fig. 3. N. sativa decreases both degranulation and increased number of mast cells induced by T. terreum. (A) While T. terreum increased the percent of degranulated mast cells in dura mater $(* p<0.05)$, R. condensata,

L. salmonicolor, and

$L$. piperatus did not change it, and $N$. sativa decreased the increase in the percent of degranulated mast cells induced by $T$. terreum $\left({ }^{*} p<0.05\right)$. (B) While

$T$. terreum increased the total mast cell numbers in dura mater $(* p<0.05)$,

$R$. condensata, $L$. salmonicolor, and

L. piperatus did not change it, and $N$. sativa decreased the $T$. terreum-induced increase in total mast cell numbers $\left({ }^{*} p<0.05\right)$. Ter: terreum group; Nig + Ter: group applied N. sativa and T. terreum; Con: condensata group; Sal: salmonicolor group; Pip: piperatus group 


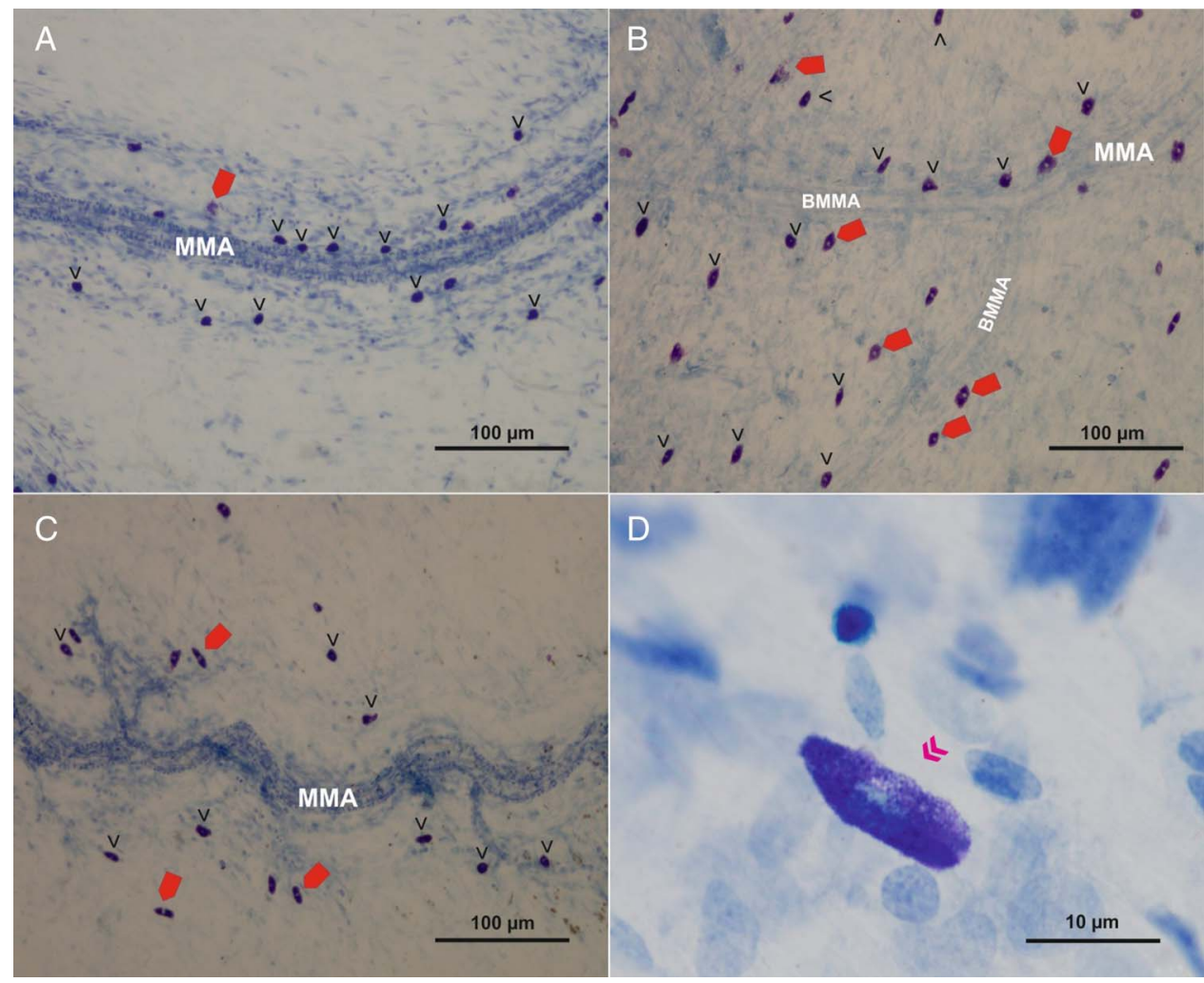

Fig. 4. The effects of $N$. sativa on degranulation states and increased number of dural mast cells induced by T. terreum. (A) Mast cells in the control group, 10× magnification, (B) T. terreum increased mild degranulation and number of mast cells, $10 \times$ magnification, (C) $N$. sativa almost completely prevented the degranulation of mast cells and decreased their numbers induced by $T$. terreum, 10× magnification, and (D) a partial degranulated mast cell with $100 \times$ magnification in the Nigella + Terreum group, $N$. sativa prevented exact degranulation of mast cells. Open arrowheads show intact mast cells and solid arrowheads show degranulated mast cells, and double open arrowhead shows partial degranulated mast cell. The increased number of mast cells in (B) compared with the control group and the decrease in (C) compared with the Terreum group. MMA: middle meningeal artery; BMMA: branch of middle meningeal artery

$(17.95 \pm 0.57 \%, p<0.05$; Figs $3 \mathrm{~A}$ and $4 \mathrm{~B}, \mathrm{C})$, moreover, total mast cell numbers in the dura of rats in the Nigella + Terreum group were also significantly less $(495 \pm 3.74, p<0.05$; Fig. 3B) than in rats in the Terreum group $(557 \pm 1.44, p<0.05)$.

\section{Discussion}

By investigating the effects of the ethanolic extract of $N$. sativa seeds and certain species of fungi including $R$. condensata, L. salmonicolor, L. piperatus, and T. terreum on the number and granulation/degranulation density of dura mater mast cells in rats, we found that among the extracts of fungi, only the extract of $T$. terreum caused significant degranulation of dural mast cells and an increase in the dural mast cell number when applied alone. On the other hand, the extract of $N$. sativa seeds inhibited dural mast cell degranulation induced by both 
the compound 48/80 and the extract of T. terreum. Moreover, it decreased the number of dural mast cells increased by the extract of $T$. terreum.

Mast cells play a vital role in the inflammatory processes including also headaches and contain three main categories of inflammatory mediators including preformed mediators stored in their secretory granules, cytokines, and lipid-derived eicosanoids (20). When mast cells are activated with antigen or synthetic chemicals, such as compound $48 / 80$, they release the mediators to their resident environment. Release of vasoactive mediators - such as histamine, vasoactive intestinal peptide, CGRP, and SP - increases vascular permeability and local blood flow in dura mater that causes plasma protein extravasation responsible for sterile neurogenic inflammation.

There are a lot of antigens in the food of plant and animal origin which induce IgE-mediated or IgE-independent reactions such as systemic allergies, inflammation, and mast cell degranulation following ingestion. There are conflicting data on whether fungi or their products cause degranulation of mast cells. In a study, it was showed that Aspergillus fumigatus hyphae induced pulmonary mast cell degranulation in the absence of IgE, an IgE-independent manner in vitro (22). In contrast to this report, it was demonstrated that the extract of Pleurotus eryngii, an edible mushroom native to Europe, inhibited release of allergic mediators containing hexosaminidase and histamine from antigen-stimulated mast cells (11).

In this study, we first showed that a fungus, T. terreum, caused massive degranulation of mast cells and increased the number of mast cells in the dura mater in rats. It was suggested that migraine is related to vasodilation resulting from neurogenic inflammation (8). Neuropeptides such as SP and CGRP, released from trigeminal sensory nerve terminals innervating meninges, cause degranulation of resident mast cells in dura mater, and thus degranulated mast cells release pronociceptive and vasoactive substances, such as ATP, histamine, bradykinin, SP, and CGRP, which activate or sensitize nociceptors in the meninges (25). Thus, mast cell degranulation is an important contributor to neurogenic inflammation underlying the migraine pathophysiology (15).

In this study, mushrooms including $R$. condensata, L. salmonicolor, L. piperatus, and T. terreum are naturally grown in the region of west Black Sea in Turkey and harvested and consumed by native population who live in the region. Some people who had consumed these fungi were hospitalized for vomiting, dizziness, and headache complaints. Our results suggested that $T$. terreum following ingestion can contribute to a headache like migraine via dural mast cell degranulation. However, to determine the ingredients of $T$. terreum that induced degranulation of dural mast cells, further studies are needed. Compound 48/80, a mixed polymer, is a mast cell degranulating agent and widely used for IgE-independent activation of mast cells (19). In this study, we used the compound 48/80 as positive control to degranulate dural mast cells, thus compound 48/80 induced massive degranulation of dural mast cells; moreover, the ethanolic extract of $N$. sativa seeds inhibited the degranulation of dural mast cells induced by both the compound 48/80 and the extract of T. terreum.

It has been reported that $N$. sativa has a lot of therapeutic properties, such as analgesic, anti-inflammatory, and antihistaminic (1). In a study, ethanolic extract of $N$. sativa seeds has been found to reduce inflammatory responses in formalin-induced inflammation in rats (7). It has been demonstrated that ethanolic extract of $N$. sativa seeds exerted an analgesic effect on acetic acid evoked nociceptive response in mice (6). Those studies explicitly have shown that the extract of $N$. sativa exhibited analgesic and anti-inflammatory effects but the mechanism of these therapeutic effects has remained unclear. Therefore, our findings suggest that 
$N$ sativa may exert its analgesic and anti-inflammatory effects by stabilizing dural mast cells. However, it has to be explored which ingredients of $N$. sativa ensure these beneficial effects.

In a study with ethanol-induced gastric mucosal damage in rats, it was showed that $N$ sativa and thymoquinone application by gavage decreased increased mast cell numbers and histamine levels in gastric tissue damaged by absolute alcohol (13), but they did not examine degranulation states of mast cells in the gastric tissue. In line with this study, we found that the extract of $N$. sativa not only decreased dural mast cell numbers increased by the extract of T. terreum but also prevented degranulation of dural mast cells induced by both the compound $48 / 80$ and the extract of $T$. terreum. Therefore, our findings can fill a gap associated with the effects of $N$. sativa on the degranulation of mast cells in dura mater. In another in vitro study with rat peritoneal mast cells, it was demonstrated that nigellone, a carbonyl polymer of thymoquinone, at low concentration inhibited histamine release induced by the secretagogues including compound 48/80, antigen in sensitized cells and the calcium ionophore A23187 (9). Consistent with this studies, in this study, our findings showed that extract of $N$. sativa seeds inhibited degranulation of dural mast cells induced by both the compound 48/80 and $T$. terreum, and moreover, it decreased the number of mast cells increased by $T$. terreum. Those studies support our findings concerning the stabilizing effect of $N$. sativa seeds on the dural mast cells. Taken together, the findings of this study or those of others show that $N$. sativa may probably exert its stabilizing effect on the mast cells via thymoquinone, the active ingredient of the seeds, but there is a need for further studies to reveal the mechanisms responsible for the stabilizing effect of $N$. sativa seeds on dural mast cells.

\section{Acknowledgement}

We would like to thank Dr. Arzu from Faculty of Agriculture and Natural Sciences, Abant Izzet Baysal University for providing the fresh extract of $N$. sativa seeds as a gift.

\section{REFERENCES}

1. Ahmad A, Husain A, Mujeeb M, Khan SA, Najmi AK, Siddique NA, Damanhouri ZA, Anwar F: A review on therapeutic potential of Nigella sativa: a miracle herb. Asian Pac. J. Trop. Biomed. 3, 337-352 (2013)

2. Al-Ghamdi MS: The anti-inflammatory, analgesic and antipyretic activity of Nigella sativa. J. Ethnopharmacol. 76, 45-48 (2001)

3. Amin B, Hosseinzadeh H: Black cumin (Nigella sativa) and its active constituent, thymoquinone: an overview on the analgesic and anti-inflammatory effects. Planta Med. 82, 8-16 (2016)

4. Athanasakis G, Aligiannis N, Gonou-Zagou Z, Skaltsounis AL, Fokialakis N: Antioxidant properties of the wild edible mushroom Lactarius salmonicolor. J. Med. Food 16, 760-764 (2013)

5. Balaha MF, Tanaka H, Yamashita H, Abdel Rahman MN, Inagaki N: Oral Nigella sativa oil ameliorates ovalbumin-induced bronchial asthma in mice. Int. Immunopharmacol. 14, 224-231 (2012)

6. Bashir MU, Qureshi HJ: Analgesic effect of Nigella sativa seeds extract on experimentally induced pain in albino mice. J. Coll. Physicians Surg. Pak. 20, 464-467 (2010)

7. Bashir MU, Qureshi HJ, Saleem T: Comparison of anti-inflammatory activity of Nigella sativa and diclofenac sodium in albino rats. J. Ayub Med. Coll. Abbottabad 27, 523-526 (2015)

8. Bozoghlanian M, Vasudevan S: Migraine pathophysiology. Future Med. 1, 337-352 (2011)

9. Chakravarty N: Inhibition of histamine release from mast cells by nigellone. Ann. Allergy 70, 237-242 (1993)

10. Fathy M, Nikaido T: In vivo modulation of iNOS pathway in hepatocellular carcinoma by Nigella sativa. Environ. Health Prev. Med. 18, 377-385 (2013)

11. Han EH, Hwang YP, Kim HG, Choi JH, Im JH, Yang JH, Lee HU, Chun SS, Chung YC, Jeong HG: Inhibitory effect of Pleurotus eryngii extracts on the activities of allergic mediators in antigen-stimulated mast cells. Food Chem. Toxicol. 49, 1416-1425 (2011) 
12. Héron A, Dubayle D: A focus on mast cells and pain. J. Neuroimmunol. 264, 1-7 (2013)

13. Kanter M, Coskun O, Uysal $\mathrm{H}$ : The antioxidative and antihistaminic effect of Nigella sativa and its major constituent, thymoquinone on ethanol-induced gastric mucosal damage. Arch. Toxicol. 80, 217-224 (2006)

14. Kilinc E, Firat T, Tore F, Kiyan A, Kukner A, Tunçel N: Vasoactive intestinal peptide modulates c-Fos activity in the trigeminal nucleus and dura mater mast cells in sympathectomized rats. J. Neurosci. Res. 93, 644-650 (2015)

15. Levy D, Burstein R, Kainz V, Jakubowski M, Strassman AM: Mast cell degranulation activates a pain pathway underlying migraine headache. Pain 130, 166-176 (2007)

16. Ozen T, Darcan C, Aktop O, Turkekul I: Screening of antioxidant, antimicrobial activities and chemical contents of edible mushrooms wildly grown in the black sea region of Turkey. Comb. Chem. High Throughput Screen. 14, 72-84 (2011)

17. Rivera-Mariani FE, Nazario-Jiménez S, López-Malpica F, Bolaños-Rosero B: Skin test reactivity of allergic subjects to basidiomycetes' crude extracts in a tropical environment. Med. Mycol. 49, 887-891 (2011)

18. Sadi G, Kaya A, Yalcin HA, Emsen B, Kocabas A, Kartal DI, Altay A: Wild edible mushrooms from Turkey as possible anticancer agents on HepG2 cells together with their antioxidant and antimicrobial properties. Int. J. Med. Mushrooms 18, 83-95 (2016)

19. Schemann M, Kugler EM, Buhner S, Eastwood C, Donovan J, Jiang W, Grundy D: The mast cell degranulator compound 48/80 directly activates neurons. PLoS One 7, e52104 (2012)

20. Thomas PS: Tumour necrosis factor-alpha: the role of this multifunctional cytokine in asthma. Immunol. Cell Biol. 79, 132-140 (2001)

21. Tore F, Tuncel N: Mast Cells: target and source of neuropeptides. Curr. Pharm. Des. 15, 3433-3445 (2009)

22. Urb M, Pouliot P, Gravelat FN, Olivier M, Sheppard DC: Aspergillus fumigatus induces immunoglobulin E-independent mast cell degranulation. J. Infect. Dis. 200, 464-472 (2009)

23. Vafaee F, Hosseini M, Hassanzadeh Z, Edalatmanesh MA, Sadeghnia HR, Seghatoleslam M, Mousavi SM, Amani A, Shafeia MN: The effects of Nigella sativa hydro-alcoholic extract on memory and brain tissues oxidative damage after repeated seizures in rats. Iran. J. Pharm. Res. 14, 547-557 (2015)

24. Zeynalov Y (2008): Medicinal Plants, Historical Development and Applications. Aden, Istanbul

25. Zhang XC, Strassman AM, Burstein R, Levy D: Sensitization and activation of intracranial meningeal nociceptors by mast cell mediators. J. Pharmacol. Exp. Ther. 322, 806-812 (2007) 